\title{
artigo
}

\section{Análise de soroconversão pós vacina hepatite B em ribeirinhos do município de Mocajuba, Pará, Brasill}

\author{
Analysis of post vaccine hepatitis soroconversion in ribeirinhos in the municipality of Mocajuba, Pará, Brazil \\ Análisis de seroconversión después de la vacuna contra la hepatitis B en costillas del municipio de Mocajuba, Pará, Brasil
}

\section{RESUMO}

Objetivo: Avaliar a soroprevalência de anticorpos anti-HBs em população ribeirinha da comunidade São Pedro do Vizeu, do Município de Mocajuba, Estado do Pará, Brasil. Método: Pesquisa de campo, descritiva, exploratória, quantitativa, de corte transversal, realizada com membros das 25 famílias residentes na Comunidade São Pedro do Vizeu, Município de Mocajuba, Pará, entre agosto de 2018 e julho de 2019. Foi coletado cerca de $16 \mathrm{~mL}$ de sangue de cada participante, e realizada os testes sorológicos por técnica imunoenzimática. Resultado: Participaram da pesquisa 133 indivíduos que apresentaram a carteira de vacinação contra hepatite $B$, destes $61,7 \%$ apresentaram o marcador anti-HBs+ isolado, demonstrando o perfil de imunidade vacinal para o VHB. Conclusão: Na população estudada, 38,3\% tomaram a vacina, porém não apresentaram níveis de titulação suficiente e estão suscetíveis a infecção. Inferiu-se que a vacina, quando utilizada corretamente, é a principal ferramenta capaz de reduzir os índices de casos confirmados e mortalidade.

DESCRITORES: Hepatite B; Vacinação; Epidemiologia; Soroconversão.

\section{ABSTRACT}

Objective: To evaluate the seroprevalence of anti-HBs antibodies in a riverside population of the São Pedro do Vizeu community, in the municipality of Mocajuba, Pará State, Brazil. Method: Field research, descriptive, exploratory, quantitative, cross-sectional, conducted with members of the 25 families living in the São Pedro do Vizeu Community, Mocajuba Municipality, Pará, between August 2018 and July 2019. Approximately $16 \mathrm{~mL}$ of blood was collected from each participant, and serological tests were performed by immunoenzymatic technique. Result: The study participants 133 individuals who presented the hepatitis B vaccination card, of which $61.7 \%$ had the anti-HBs+ marker isolated, demonstrating the profile of vaccine immunity for HBV. Conclusion: In the population studied, 38.3\% took the vaccine, but did not have sufficient titration levels and are susceptible to infection. It was inferred that the vaccine, when used correctly, is the main tool capable of reducing the rates of confirmed cases and mortality.

DESCRIPTORS: Hepatitis B; Vaccination; Epidemiology, Seroconversão.

\section{RESUMEN}

Objetivo: Evaluar la seroprevalencia de anticuerpos anti-HBs en una población ribereña de la comunidad de San Pedro do Vizeu, en el municipio de Mocajuba, Estado de Pará, Brasil. Método: Investigación de campo, descriptiva, exploratoria, cuantitativa, transversal, realizada con miembros de las 25 familias que viven en la Comunidad de San Pedro do Vizeu, Municipio de Mocajuba, Pará, entre agosto de 2018 y julio de 2019. Se recogieron aproximadamente 16 ml de sangre de cada participante, y se realizaron pruebas serológicas mediante una técnica inmunoenzimática. Resultado: El estudio participa 133 personas que presentaron la tarjeta de vacunación contra la hepatitis B, de las cuales el 61,7\% tenían aislado el marcador anti-HBs+, lo que demuestra el perfil de inmunidad vacuna para el VHB. Conclusión: En la población estudiada, el 38,3\% tomó la vacuna, pero no tenía niveles de valoración suficientes y son susceptibles a la infección. Se dedujo que la vacuna, cuando se utiliza correctamente, es la principal herramienta capaz de reducir las tasas de casos confirmados y mortalidad.

DESCRIPTORES: Hepatitis B; Vacunación; Epidemiología, Seroconversión.

RECEBIDO EM: 19/01/2021 APROVADO EM: 27/01/2021

\section{Letícia Gomes de Oliveira}

Enfermeira, Especialista em Microbiologia e Imunologia, Mestranda em Epidemiologia e Vigilância em Saúde pelo Instituto Evandro Chagas/ Secretaria de Vigilância em Saúde/ Ministério da Saúde.

ORCID: 0000-0002-8830-728X 


\section{Candida Maria Abrahão de Oliveira}

Enfermeira, Doutora em Virologia pelo Instituto Evandro Chagas/ Secretaria de Vigilância em Saúde/ Ministério da Saúde. ORCID: 0000-0001-6253-7477

\section{Dickson Ciro Nascimento de Brito}

Biomédico, Mestrando em Epidemiologia e Vigilância em Saúde pelo Instituto Evandro Chagas/ Secretaria de Vigilância em Saúde/ Ministério da Saúde.

ORCID: 0000-0001-8389-3980

\section{Fabiolla da Silva dos Santos}

Biomédica, Doutora em Virologia pelo Instituto Evandro Chagas/ Secretaria de Vigilância em Saúde/ Ministério da Saúde, Coordenadora da Pós graduação em Microbiologia e Imunologia da Escola Superior da Amazônia.

ORCID: 0000-0001-6317-0988

\section{Heloisa Marceliano Nunes}

Médica, Doutora em Biologia de Doenças Infecciosas e Parasitárias, Chefe da Seção de Hepatologia do Instituto Evandro Chagas/Secretaria de Vigilância em Saúde/ Ministério da Saúde.

ORCID: 0000-0002-9335-6932

\section{INTRODUÇÃO}

A s infecções pelo vírus da hepatite B (VHB) constituem um sério problema de saúde pública global. Segundo a Organização Mundial de Saúde (OMS) existem aproximadamente dois bilhões de infectados pelo $\mathrm{VHB}$ em todo o mundo ${ }^{(1)}$. No período de 1999 a 2018, foram notificados 233.027 casos de hepatite B no Brasil, com 6,7 casos para cada 100 mil habitantes, em 2018. As taxas de detecção das regiões Sul e Norte têm se mostrado superiores à taxa nacional, sendo a hepatite $B$ de elevada prevalência em populações quilombolas, indígenas e ribeirinhas ${ }^{(2,3)}$.

Existem testes rápidos disponíveis no Sistema Único de Saúde (SUS) para a detecção da infecção pelo VHB, ao qual preconiza testar todas as pessoas ao menos uma vez na vida, sendo que populações em vulnerabilidade precisam testar periodicamente. Os medicamentos utilizados para o tratamento da hepatite $B$ não garantam a cura da doença, porém ela é imunoprevenível podendo a vacina ser aplicada em qualquer faixa etária, independente de condições de vulnerabilidade ${ }^{(2,4)}$.

As vacinas contra o VHB estão disponíveis em formulações monovalentes que protegem somente contra hepatite B e, também, combinadas que protegem contra o VHB e outras doenças ${ }^{(5)}$. O esquema padrão de vacinação inclui um $\mathrm{mL}$, com 20 mg de HBsAg recombinante administrado em três doses ${ }^{(6)}$. A segunda dose deve ser administrada 2 semanas após a primeira, a partir dessa dose já existe resposta, mas para uma proteção a longo prazo, uma terceira deve ser aplicada a partir de seis meses após

\section{As infecções pelo}

vírus da hepatite $B$

(VHB) constituem

um sério problema

de saúde pública

global. Segundo

a Organização

Mundial de Saúde

(OMS) existem

aproximadamente

dois bilhões de

infectados pelo VHB

em todo o mundo a primeira dose. Quando não ocorre soroconversão o indivíduo deverá repetir o esquema vacinal. No caso de imunodepressão, o segundo esquema deve ser preferencialmente aplicado na dose de dois $\mathrm{mL}^{(7)}$.

A vacinação é o ato de aplicar a vacina e a imunização é o desenvolvimento de anticorpos específicos derivados da vacina aplicada, existindo um mecanismo que avalia a efetividade da vacina contra hepatite B por meio do teste sorológico anti-HBs, contudo, diferente da vacina, o exame não é disponibilizado na rede pública de saúde, como rotina, após a vacinação ${ }^{(8)}$.

Considerando as diversas formas de transmissão da infecção pelo VHB e a prevalência mais elevada da infecção, em populações de maior vulnerabilidade, como ribeirinhos ${ }^{(9)}$, está pesquisa buscou responder: "Qual a taxa de soroconversão da vacina contra Hepatite B, na população ribeirinha do Município de Mocajuba, Pará?”. Deste modo, este trabalho objetivou avaliar a soroprevalência de anticorpos anti-HBs em população ribeirinha da comunidade São Pedro do Vizeu, do Município de Mocajuba, Estado do Pará, Brasil.

\section{METODO}

Trata-se de uma pesquisa de campo, descritiva, exploratória, quantitativa, de corte transversal, desenvolvida na Comunidade São Pedro do Vizeu, Município de 


\section{artigo}

Oliveira, L.GL.; Oliveria, C.M.A.; Brito, D.C.N.; Santos, F.S.; Nunes, H.M.;

Análise de soroconversão pós vacina hepatite B em ribeirinhos do município de Mocajuba, Pará, Brasil

Mocajuba, Pará, entre agosto de 2018 e julho de 2019, aprovada pelo Comitê de

Ética em Pesquisa com Seres Humanos, do Instituto Evandro Chagas (CEP/IEC)

Tabela 1 - Distribuição da população segundo faixa etária e sexo,

Comunidade São Pedro do Vizeu, Mocajuba, Pará, Brasil, outubro de 2018.

$\begin{array}{lcccccc}\begin{array}{l}\text { FAIXA ETÁRIA } \\ \text { (ANOS) }\end{array} & \begin{array}{c}\text { POPULAÇÃO } \\ \text { EXAMINADA }\end{array} & \% & \text { MASCULINO } & \% & \text { FEMININO } & \% \\ <1 & 2 & 1,5 & - & - & 2 & 100 \\ 1 \text { a } 9 \text { a } & 39 & 29,3 & 22 & 56,4 & 17 & 43,6 \\ 10 \text { a } 19 & 41 & 30,8 & 18 & 43,9 & 23 & 56,1 \\ 20 \text { a } 29 & 18 & 15,5 & 7 & 38,9 & 11 & 51,1 \\ \text { 30 a 39 } & 11 & 8,3 & 2 & 18,2 & 9 & 81,8 \\ 40 \text { a 49 } & 11 & 8,3 & 3 & 27,3 & 8 & 72,7 \\ 50 \text { a 59 } & 9 & 5,7 & 3 & 33,3 & 6 & 66,7 \\ \geq 60 & & & & & & \\ 2 & 1,5 & 1 & 50,0 & 1 & 50,0 & \\ \text { Total } & 133 & 100,0 & 56 & 42,1 & 77 & 57,9\end{array}$

Fonte: Pesquisa de campo, outubro de 2018; - = dado numérico igual a zero, não resultante de arredondamento

Tabela 2 - Perfil dos participantes do estudo, segundo ocupação habitual, escolaridade e estado civil, Comunidade São Pedro do Vizeu, Mocajuba,

Pará, Brasil, outubro de 2018.

\begin{tabular}{|c|c|c|c|}
\hline \multicolumn{2}{|c|}{ VARIÁVEIS } & PARTICIPANTES (N=133) & $\%$ \\
\hline \multirow{7}{*}{ Ocupação habitual } & Estudante & 78 & 58,6 \\
\hline & Pescador & 28 & 21,0 \\
\hline & Agricultor & 11 & 8,4 \\
\hline & Menor (sem ocupação) & 6 & 4,5 \\
\hline & Professor & 2 & 3,8 \\
\hline & Aposentado & 3 & 2,2 \\
\hline & Do lar & 3 & 2,2 \\
\hline \multirow{10}{*}{ Escolaridade } & Funcionário público & 1 & 0,7 \\
\hline & Fundamental incompleto & 98 & 73,7 \\
\hline & Médio incompleto & 11 & 8,4 \\
\hline & Não se aplica & 6 & 4,5 \\
\hline & Analfabeto & 4 & 3,0 \\
\hline & Médio completo & 4 & 3,0 \\
\hline & Maternal & 4 & 3,0 \\
\hline & Superior incompleto & 3 & 2,2 \\
\hline & Superior completo & 2 & 1,5 \\
\hline & Fundamental completo & 1 & 0,7 \\
\hline \multirow{3}{*}{ Estado Civil } & Solteiro & 94 & 70,7 \\
\hline & Casado & 37 & 27,8 \\
\hline & Viúvo & 2 & 1,5 \\
\hline
\end{tabular}

sob Parecer de No 2.736 .328 e CAEE 90604418.4.0000.0019.

Foram incluídos no estudo membros das 25 famílias residentes nessa comunidade, de qualquer faixa etária e sexo, presentes no momento da coleta de dados e amostras, que já haviam recebido a vacina contra hepatite $\mathrm{B}$, comprovada pela carteira de vacinação e foram excluídos os indivíduos não vacinados e não residentes na comunidade.

Para esclarecimentos sobre a pesquisa, foi realizada a leitura e assinatura do Termo de Consentimento Livre e Esclarecido e aos menores de 18 anos o Termo de Assentimento Livre e Esclarecido. Em seguida foi aplicado um questionário que avaliou: sexo, idade, estado civil, ocupação, grau de escolaridade, situação vacinal contra o VHB, resposta sorológica e fatores que dificultavam o acesso a vacina.

Foi coletado cerca de $16 \mathrm{~mL}$ de sangue de cada participante, por punção intravenosa, as amostras foram centrifugadas para separação do soro. Os testes sorológicos foram realizados por técnica imunoenzimática (ELISA) obedecendo as recomendações do fabricante.

As informaçốes coletadas foram armazenadas em uma base de dados no Programa EPIINFO 2007, versão 7.1.0.6. e no programa Microsoft Office Excel 2007, que posteriormente, foram exportados para o Programa Bioestat 5.0 ${ }^{(10)}$. Para as análises estatísticas utilizou-se o teste qui-quadrado. Foram calculadas a média e a mediana para as variáveis quantitativas, devido sua praticidade e aplicabilidade em diversas áreas, com nível de confiança de 95\%.

\section{RESULTADOS}

Participaram da pesquisa 133 indivíduos sendo $57,9 \%(77 / 133)$ do sexo feminino. $\mathrm{Na}$ população examinada a maior frequência estava na faixa etária de 10 a 19 anos, o que correspondeu a $30,8 \%$ $(41 / 133)$ e a menor frequência entre os menores de um ano $1,5 \%(2 / 133)$ e maiores de 60 anos em 1,5\% (2/133), conforme apresentado na tabela 1 . A média de idade da população foi de 20,6 (variação de 3 meses a 87 anos) e a mediana de 13 anos. 
Figura 1 - Distribuição dos fatores que dificultam o acesso à vacina da hepatite B entre os participantes da Comunidade São Pedro do Vizeu, Mocajuba, Pará, Brasil, outubro de 2018

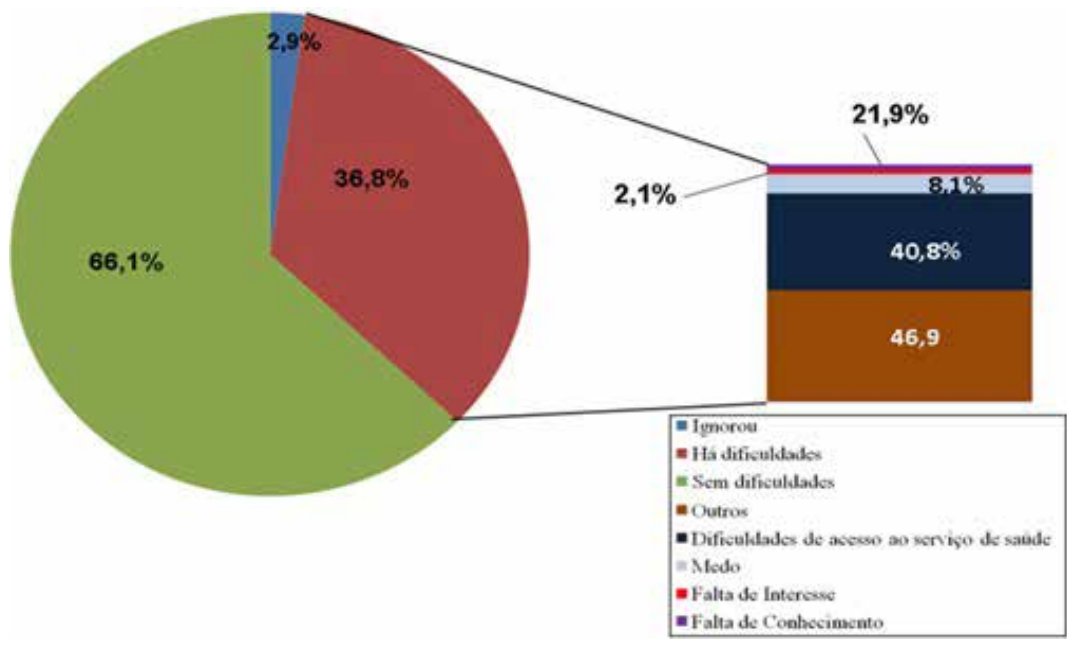

Fonte: Pesquisa de campo, outubro de 2018

Tabela 3 - Níveis de anticorpos anti-HBs produzidos após a vacinação nos participantes da Comunidade São Pedro do Vizeu, Mocajuba, Pará, Brasil, outubro de 2018

$\begin{array}{ccc}\text { QUANTIFICAÇÃO ANTI-HBS MUI/ML } & \text { N } & \% \\ <10 & 51 & 38,3 \\ 10-100 & 44 & 33,7 \\ 101-200 & 14 & 10,4 \\ 201-300 & 5 & 3,6 \\ 301-400 & 7 & 5,1 \\ 401-499 & 1 & 0,7 \\ >500 & 11 & 8,2 \\ \text { Total } & 133 & 100,0\end{array}$

Em relação às variáveis quanto à ocupação habitual 57,1\% (81/133) eram estudantes e $21 \%$ (29/133) eram pescadores; quanto à escolaridade $73,7 \%(98 / 133)$ possuíam ensino fundamental incompleto; quanto ao estado civil, observou-se que $70,7 \%$ (94/133) eram indivíduos solteiros, conforme apresentado na tabela 2.

Quando questionados a respeito das barreiras ao acesso à vacina da hepatite B, 66,1\% (88/133) afirmaram não possui qualquer tipo de dificuldade. Em contrapartida, 36,8\% (49/133) relataram ter dificuldades para tomar a vacina, dentre as razões mais citadas foram o acesso aos serviços de saúde em $40,8 \%$ (20/49) e o medo em tomar a vacina em 8,1\% (4/49), conforme apresentado na figura 1.

Entre os 133 indivíduos que apresentaram registro na carteira de vacinação de aplicação da vacina contra hepatite $B, 61,7 \%(82 / 133)$ apresentaram o marcador anti-HBs+ isolado, demonstrando o perfil de imunidade vacinal para o VHB, logo 38,3\% (51/133) apresentaram níveis de titulação abaixo do limite considerado imune $(10 \mathrm{mUI} / \mathrm{mL})$, significando que não houve soroconversão e que os mesmos estavam suscetíveis a infecção, conforme ilustrado na tabela 3.

Entre os participantes do estudo, $21,8 \%$ $(29 / 133)$ apresentaram a carteira de vacinação com as três doses da vacina, preconizadas pelo Ministério da Saúde, entre esses $37,9 \%(11 / 29)$ desenvolveram títulos protetores do marcador sorológico anti-HBs após a completude do esquema vacinal. Identificou-se por meio da carteira de vaci-

Tabela 4 - Distribuição do número de doses de vacinas aplicadas e a soroconversão para anti-HBs isolado entre os participantes da Comunidade São Pedro do Vizeu, Mocajuba, Pará, Brasil, outubro de 2018

\begin{tabular}{|c|c|c|c|c|c|c|c|c|c|}
\hline \multirow[b]{2}{*}{ FAIXA ETÁRIA (ANOS) } & \multirow[b]{2}{*}{$\mathbf{N}$} & \multicolumn{8}{|c|}{$\mathrm{N}^{\circ}$ DE DOSES DE VACINA APLICADAS E SOROCONVERSÃO PARA ANTI-HBS } \\
\hline & & 1 & ANTI-HBS+ (\%) & 2 & ANTI-HBS+ (\%) & 3 & ANTI-HBS+ (\%) & $\begin{array}{c}\mathbf{N}^{\circ} \text { DOSES } \\
\text { DESCONHECIDAS }\end{array}$ & ANTI-HBS+ ( $\%)$ \\
\hline$<1$ & 2 & - & - & 1 & $0(0,0)$ & - & - & 1 & $1(100,0)$ \\
\hline 01-09 & 39 & - & - & 1 & $0(0,0)$ & 6 & $3(50,0)$ & 32 & $17(53,1)$ \\
\hline $10-19$ & 41 & - & - & 1 & $1(100,0)$ & 16 & $6(37,5)$ & 24 & $13(54,1)$ \\
\hline $20-29$ & 18 & 1 & $1(100,0)$ & 1 & $1(100,0)$ & 4 & $2(50,0)$ & 12 & $11(91,7)$ \\
\hline $30-39$ & 11 & - & - & - & - & 1 & $0(0,0)$ & 10 & $10(100,0)$ \\
\hline $40-49$ & 11 & - & - & - & - & 2 & $0(0,0)$ & 9 & $7(77,8)$ \\
\hline $50-59$ & 9 & - & - & - & - & - & - & 9 & $8(88,9)$ \\
\hline
\end{tabular}




$\begin{array}{lccccccccc}\geq 60 & 2 & - & - & - & - & - & - & 2 & 2(100,0) \\ \text { Total } & 133 & 1 & 1(100,0) & 4 & 2(50,0) & 29 & 11(37,9) & 99 & 69(69,6)\end{array}$

Fonte: SAHEP, pesquisa de campo, 2018; N= população examinada; - = dado numérico igual a zero, não resultante de arredondamento; anti-HBs+ isolado = perfil compatível com proteção vacinal.

nação, que uma pessoa tomou apenas uma dose de vacina e conseguiu a soroconversão para anti-HBs e que quatro pessoas foram vacinadas com duas doses da vacina, com $50 \%$ (2/4) de soroconversão. Identificou-se também que 69,6\% (69/99) dos indivíduos testados para o anti-HBs não tinham informações sobre o número de doses de vacina aplicadas (Tabela 4).

\section{DISCUSSÃO}

$\mathrm{O}$ grande desafio relacionado à vacinação contra hepatite $\mathrm{B}$ na comunidade estudada foi a baixa adesão à conclusão do esquema vacinal. Estudos realizados por diversos pesquisadores mostraram que a completude vacinal de três doses contra hepatite B tem sido difícil em populações de maior vulnerabilidade ${ }^{(11,12,13)}$.

Considerando que as condições de vida das populações ribeirinhas, que vivem afastadas dos principais centros urbanos e tem como principal meio de locomoção o rio, ter acesso a vacina parece um ideal distante, o estudo mostrou as dificuldades em completar o esquema convencional contra hepatite $\mathrm{B}$, configurando-se como um desafio remanescente para a prevenção do $\mathrm{VHB}^{(14)}$.

$\mathrm{A}$ ampliação da vacinação contra o VHB pode ser uma estratégia para melhorar a cobertura vacinal em populações de maior vulnerabilidade ${ }^{(15,16)}$. Dessa forma, a implementação do esquema acelerado contra hepatite $\mathrm{B}$ parece uma promissora estratégia de imunização de populações vulneráveis.

Vários fatores podem estar associados a falta de soroconversão do anti-HBs, como armazenamento inadequado da vacina, administração fora do recomendado, a idade, o índice de massa corporal, cirrose ou insuficiência renal crônica, imunossupressão, transplantados, hemodiálise crônica, diabetes tipo I, alcoolismo e tabagismo ou infecções no momento da vacinação ${ }^{(17,18)}$.

A vacinação contra o $\mathrm{VHB}$ é uma estratégia comprovada e bem estabelecida para a prevenção de doenças. De acordo com a
$\mathrm{CDC}^{(19)}$ a vacina contra o VHB na população em geral confere imunidade a mais de 90\% dos indivíduos vacinados. Neste estudo, foi possível notar que 38,3\% receberam a vacina, porém apresentaram níveis de titulação abaixo do limite considerado imune $(10 \mathrm{mUI} / \mathrm{mL})$, porcentagem acima do que é estimado por diversos estudos que apontam cerca de $5 \%$ a $10 \%$ de irresponsividade após a completude do esquema vacinal ${ }^{(20,21)}$.

\section{A ampliação da}

vacinação contra

o VHB pode ser

uma estratégia

para melhorar a

cobertura vacinal em

populações de maior

vulnerabilidade.

Dessa forma, a

implementação do

esquema acelerado

contra hepatite

$B$ parece uma

promissora estratégia

de imunização

de populações

vulneráveis.
Foi possível identificar que os maiores de 60 anos responderam a vacina diferente dos estudos de Locarrini et al., (2015) que afirma que as pessoas idosas não são tão responsivas quanto as crianças ou adolescentes ${ }^{(22)}$.

Nota-se ainda, a baixa adesão da completude da vacina, onde não foi possível identificar em qual fase de aplicação estava a dosagem. Pode estar associado ao intervalo e sugere um esquema vacinal acelerado contra hepatite $\mathrm{B}$ de 0,7 e 21 dias, para populações vulneráveis, se apresentando como estratégia que pode qualificar o atendimento a essa população prevenindo a infecção pelo $\mathrm{VHB}^{(23)}$.

Não há indicação para a testagem do anti-HBs na população em geral, somente é indicada para profissionais da área da saúde ${ }^{(24)}$, a criação de uma vigilância sorológica em consonância com a vigilância epidemiológica, seria um grande avanço pois seria possível rastrear as pessoas que estão imunes e não somente os casos confirmados da doença Hepatite B.

\section{CONCLUSÃO}

Pode-se inferir que a vacina é a principal ferramenta capaz de reduzir os índices de casos confirmados e a mortalidade pelo VHB, isso quando esta ferramenta é corretamente utilizada. Percebeu-se que na população ribeirinha do munícipio de Mocajuba, 38,3\% tomaram a vacina em algum momento da vida, porém apresentaram níveis de titulação abaixo do limite e estão suscetíveis a infecção.

É surpreendente como o cenário é favorável para a condição vacinal e desfavorável para condição imune na população estudada, principalmente por se tratar de uma doença imunoprevinível. Deste modo, é necessário criar estratégias para ampliar a cobertura vacinal e aumentar a imunidade, sobretudo em grupos de maior vulnerabilidade, como a população de São Pedro do Vizeu. Além disso, destaca-se a importância de capacitações contínuas dos profis- 
sionais sobre sala de vacina e transporte de imunobiológicos às populações longe de centros urbanos.

No presente estudo apenas $21,8 \%$ de indivíduos apresentaram as três doses registradas na carteira de vacinação, conside- rando-se que esse documento é o principal comprovante de que o indivíduo recebeu o esquema vacinal, sugere-se como forma de melhorar a conservação da carteira de vacinação, que ela também seja solicitada por ocasião da matrícula escolar.
E por último, quem sabe em um futuro não tão distante, poder ampliar à população em geral o acesso ao teste sorológico do marcador vacinal (anti-HBs) para confirmação da eficácia da vacinal após sua aplicação. -

\section{REFERÊNCIAS}

1. World Health Organization Hepatitis B. 2017. Disponível em: http://www.who.int/vaccines-diseases/diseases/hepatitis_b. htm. Acesso em: 28 dez 2018

2. Ministério da Saúde (BR), Secretaria de Vigilância em Saúde. Departamento de Doenças de Condições Crõnicas e Infecções sexualmente transmissiveis. 0 que são hepatites virais. [Internet]. 2020a. Disponivel em: http://www.aids.gov.br/pt-br/ publico-geral/hv/o-que-sao-hepatitesvirais\#: :text=Entretanto $\% 2$ C $\%$ 20quando $\% 20$ presentes $\% 2$ C 20elas $\% 20$ podem,v\%C3\%ADrus\%20A\%2C\%20B\%20e\%20C. Acesso em: 08 Dez. 2020.

3. Leão RNQ, Bichara CNC, Fraiha NH, Vasconcelos PFC. Medicina tropical e infectologia na Amazônia (Vol. 1). Belém: Samauma; 2013.

4. Rio Grande do Sul. Centro Estadual de Vigilância em Saúde. Núcleo de Imunizações (Org.). Instrução normativa: calendário nacional de vacinação. 2018. Disponível em: https://cevs.rs.gov. br/upload/arquivos/201802/20152253-instrucao-normativa-calendario-nacional-de-imunizacoes-2018.pdf. Acesso em: 22 out. 2018

5. Lavanchy D. Viral hepatitis: global goals for vaccination. J Clin Virol. [S. I.], v. 55, n. 4, pp. 296-302, Dec 2012.

6. Ministério da Saúde (BR). Manual de normas e procedimentos para vacinação. Brasília: Ministério da Saúde. 2014.

7. Ministério da Saúde (BR), Secretaria de Vigilância em Saúde. Coordenação-Geral de Desenvolvimento da Epidemiologia em Serviços. Guia de Vigilância Epidemiológica. v.2. Brasília, 2019.

8. Borges AM.; Sulzbacher A; Mello MCVA. Perfil epidemiológico da Hepatite B: Conhecer para prevenir. Saúde Coletiva (Barueri), n. 52, p. 2080-2093, 2020.

9. Ministério da Saúde (BR), Secretaria de Vigilância em Saúde. Departamento de DST, Aids e Hepatites Virais. Protocolo clínico e diretrizes terapêuticas para atenção integral às pessoas com infecções sexualmente transmissíveis. Brasília: Ministério da Saúde, 2015.

10. Ayres M, Ayres JRM, Ayres DL, Santos AS. BioEstat 5.0: aplicações estatísticas nas áreas das ciências biológicas e médicas. 5. ed. Belém: Sociedade Civil Mamirauá; 2007. 364 p.

11. Magalhães RLB, et al. Baixa completude da vacina contra hepatite B em mulheres profissionais do sexo. Rev Bras Enferm. [S. I.], v. 70, n. 3, pp. 514-519, maio / jun. 2017.

12. Carneiro LM, et al. Outreach hepatitis $B$ vaccination of female sex workers in centralwest Brazil: immunization status, compliance, and immune response. J Public Health Manag Pract. [S.I.], v. 20, n. 6, pp. 662-6, Nov - Dec, 2014.

13. Nyamathi $A$, et al. Correlates of hepatitis $B$ virus and HIV knowledge among gay and bisexual homeless young adults in Hollywood. Am J Mens Health, [S. I], v. 7, n. 1, pp. 18- 26, 2013.

14. Ogholikhan S, Schwaez KB. Hepatitis Vaccines. Vacines, [S. I.], v. 4, n. 1, p. 6, 2016.

15. Ministério da Saúde (BR), Secretaria de Vigilância em Saúde. Boletim epidemiológico: Hepatites Virais 2018. Brasília, DF, 2018. v. 49, n. 31.

16. Mast $E E$, et al. A comprehensive immunization strategy eliminate transmission of hepatitis B virus infection in the United States. Recommendations of the Advisory Committee on Immunization Practices (ACIP) - Part 1: Immunization of infants, children, and adolescents. MMWR Recomm Rep., [S. I.], n. 54, pp. 1-31, Dec 2005.

17. Tong HV, Bock CT, Velavan TP. Genetic insights on host and hepatitis B virus in liver diseases. Mutat Res Rev Mutat Res. v. 762, pp. 65-75, 2014.

18. Tajiri $K$, Shimizu $Y$. Unsolved problems and future perspectives of hepatitis B virus vaccination. World J Gastroenterol., [S. I.], v. 21, pp. 7074-7083, 2015.

19. Centro de Controle e Prevenção de Doenças. Epidemiologia e Prevenção de Doenças Preveníveis por Vacinas. (13a ed.). Washington DC: Fundação de Saúde Pública. 2017.

20. Souza FO. et al. Vacinação contra hepatite B e Anti-HBS entre trabalhadores da saúde. Cad Saude Colet., Rio de Janeiro, v. 23, n. 2, p. 172-179, 2015.

21. Siqueira RJ, Filardi MBS, Marziale MHP. Acidentes de trabalho com material biológico ocorridos em municípios de Minas Gerais. Rev Bras Enferm., [S. I.], v. 67, n. 1, p. 119-126, jan./ fev. 2014.

22. Locarnini S. et al. Strategies to control hepatitis B: Public policy, epidemiology, vaccine and drugs. J. Hepatol. [S. I.], v. 62, 1 Suppl, pp. S76-S86, Apr 2015.

23. Oliveira VMCD. Adesão e avaliação da soroconversão à vacina contra hepatite b em pessoas que vivem em situação de rua. Dissertação (Mestrado em Enfermagem) - Universidade Federal do Piauí, Teresina, 2019.

24. Biblioteca Virtual de Saúde. Núcleo de Telessaúde Rio Grande do Sul. Atenção Primária em Saúde. Deve-se revacinar paciente contra hepatite B quando o resultado do anti-HBs for inferior a 10 UI/mL? 2019. Disponivel em: https://aps.bvs.br/aps/deve-serevacinar-paciente-contra-hepatite-b-quando-o-resultado-doanti-hbs-for-inferior-a-10-ui-ml/. Acesso em: 08 Dez. 2020 